\title{
Über besondere symmetrische Punktsysteme zweiten Grades und Poncelet'sche Vierecke.
}

\author{
Von Karl Schober in Innsbruck.
}

Wenn in zwei conlocalen $m$-n-deutigen Elementensystemen $m=n$ wird, so bilden sie bekanntlich nach $\mathrm{Weyr}{ }^{1}$ ) ein symmetrisches Elementensystem $n^{\text {ten }}$ Grades ${ }^{2}$ ), worin jedem Elemente $n$ andere desselben Systems entsprechen.

Im Folgenden wird ein kleiner Beitrag zur Theorie der in dieser Weise definierten symmetrischen Elementensysteme geliefert; damit soll einerseits gezeigt werden, wie sich manches Bekannte von einem anderen Gesichtspunkte aus behandeln lässt, andererseits dürften die gefundenen Resultate auch einiges Neue enthalten.

Wir wollen nämlich diejenigen speciellen Punktsysteme zweiten Grades betrachten, welche auf einer Curve zweiter Ordnung durch Strahleninvolutionen ausgeschnitten werden, deren Scheitel nicht auf der Curve liegen.

1. Die Strahleninvolutionwerdezunächstalseine rechtwinklige $v$ orausgesetzt.

Es sei $C$ der gegebene Kegelschnitt und $S$ der Scheitel der Involution $J$. Irgend zwei entsprechende Strablen $a$ und $a^{\prime}$ der'selben sehneiden $C$ in den Punktepaaren $A_{1}, A_{2}$, bezw. $A_{1}{ }^{\prime}, A_{2}{ }^{\prime}$. Jedem der Schnittpunkte eines Strahles entsprechen die beiden

1) Die Theorie der mehrdentigen geometrischen Elementargebilde ( $m$ - $n$-deu tigen und symmetrischen Elementensysteme) ist bekanntlich von dem verstorbenen Professor Wr. Emil We yr zu hoher Vollkommenheit entwickelt und mit viel Eleganz von ihm und später in analoger Woise auch von anderen*) in der Curventheorie verwertet worden. Von seinen zahlreichen dieses Gebiet betreffenden Arbeiten seien hier nur die fundamentalen Erstlingsarbeiten namentlich hervorgehoben: "Theorie der mehrdeutigen geom. Elementargebilde etc.“ Leipzig, 1869. - „Über algebraische Curven"- Sitzungsberichte d. königl. b. Ges. d. W., Prag, 1869. „Uber Punktsysteme auf rationalen Curven." Ibid., 1873. - „Beiträge zur Curvenlehre." Wien, 1880.

2) Symmetrische oder involutorische Correspondenz $[n]$ nach Sturm, der sich wiederholt auf Weyr beruft. Vergl. R, Sturm. "Die Gebilde ersten und zweiten Grades der Liniengeometrie in synthetischer Behandlung. " I. Theil, Leipzig, 1892, pag. $16 \mathrm{ff}$.

*) So z. B. in neuester Zeit von W. Binder, "Theorie der unicursalen Plancurven vierter bis dritter Ordnung." Leipzig, 1896. 
Schnittpunkte des zu ihm normalen Strahles; es constituieren so mit die Schnittpunkte aller einander entsprechenden Strahlen von $J$ auf $C$ ein symmetrisches Punktsystem zweiten Grades $\sigma$, dessen Elemente sich zu Quadrupeln gruppieren. ${ }^{1}$ ) Die Directionscurve desselben ist ein Kegelschnitt $\Gamma$; dieser berührt die Seiten der einfachen Vierecke, deren Ecken Quadrupel zusammengehöriger Punkte sind, und hat den Scheitel $S$ der Strahleninvolution $J$ zum Brennpunkte, denn die Diagonalen jedes solchen Viereckes sind zwei conjugierte und als ein zusammengehöriges Strahlenpaar von $J$ auch zueinander normale Polaren von $\Gamma$.

Damit ist auf kurzem Wege ein schon von Poncelet²) herstammender, jedoch auf wesentlich andere Art abgeleiteter Satz bewiesen worden, den wir folgendermaßen ansdrücken wollen:

Alle Sehnen eines Kegelschnittes $C$, welehe aus einembeliebigen Punkte $S$ seiner Ebene unter rechtem Winkel erscheinen, hüllen einen Kegelschnitt $\Gamma$ ein, der jenen Punkt zum Brennpunkte hat. ${ }^{3}$ )

2. Obwohl $\Gamma$ durch eines der oben genannten Vierecke bereits vollkommen bestimmt ist, soll doch noch die directe Construction der Hauptachse besprochen werden.

Da jedes dem Directionskegelschnitte $\Gamma$ umgeschriebene Viereck gleichzeitig dem Kegelschnitte $C$ eingeschrieben ist, so ist $S$ ein Eckpunkt und die Verbindungsgerade $s$ der Schnittpunkte der Gegenseiten des Viereckes die gegenüberliegende Seite des den beiden Kegelschnitten $C$ und $\Gamma$ gemeinsamen Polardreieckes und, da $S$ ein Brennpunkt von $\Gamma$ ist, weiter $s$ die entsprechende Directrix des letzteren. Es ist somit die Hauptachse von $\Gamma$ normalzur Polaren des Punktes $S$ in Bezug a uf $C$.

Da für den Kegelschnitt $C$ die Richtung von $s$ conjugiert ist derjenigen des durch $S$ gehenden Durchmessers $U T$, somit der dem letzteren conjugierte $V W$ parallel $s$ sein muss, so ist (unabhängig von s) die Hauptachse $\nabla$ on $\Gamma$ a uch normal zu demjenigen Durchmesser von $C$, der dem durch $S$ gehenden conjugiert ist.

Um die Scheitel von $\Gamma \mathrm{zu}$ erhalten, ermitteln wir dasjenige $C$ ein- und $\Gamma$ umgeschriebene Viereck, dessen eine Gegenseitenpaar zu $s$ parallel ist; dies letztere steht auf der Hauptachse normal und schneidet auf ihr die Scheitel aus. Die Ecken dieses Viereckes sind diejenigen zwei Punktepaare, welche dem Punktsystem $\sigma$ und der Involution gemeinsam sind, die auf $C$ durch die zu $s$ oder zum Durchmesser $V W$ parallelen Secanten bestimmt

1) Symmetrische Correspondenz [2]. Man kann auch sagen, in der durch das Strahlenbiischel $S$ auf $C$ erzeugten Involution werden die Punktepaare in bestimmter Weise einander als entsprechend zugeordnet.

2) "Traité des propriétés proj. des figures." Paris 1822, pag. 288.

3) Vergl, diesen Satz betreffend, Salmon-Fiedler, Mnalyt. Geometrie der Kegelschnitte." 5. Aufl.. pag. 743, und O. Richter in "Zeitschrift f. Math. und Phys." 36. Jahrg., pag. 49. 
ist. Diese zwei Punktepaare sind offenbar die Schnittpunkte der Normalstrahlen der Strahleninvolution, durch welche die zuletzt genannte Punktinvolution aus $S$ projiciert wird, mit $C$.

Auf eine andere, in ihrer Ausführung einfachere Art können die Ecken jenes Viereckes auch folgendermaßen erhalten werden. In der durch den Kegelschnitt $C$ auf $s$ bestimmten Involution ist der Schnittpnnkt der convergenten Seiten des in Rede stehenden Viereckes das Centrum $O$, da der entsprechende Punkt als Schnittpunkt der zu $s$ parallelen Gegenseiten im Unendlichen liegt; dasselbe ist aber auch der Sehnittpunkt des zu $s$ conjugierten Durchmessers von $C$ mit $s$. Wird nun um $O$ als Mittelpunkt der Kreis beschrieben, welcher durch $S$ geht, so schneidet dieser $s$ in zwei Punkten, deren Verbindungsgeraden mit $S$ aufeinander normal stehen und auf $C$ die Ecken des verlangten Viereckes ausschneiden.

Noch eine andere Ermittelung der Hauptachse von $\Gamma$ ist später in Art. 12 besprochen.

3. Alle durch Quadrupel zusammengehöriger Punkte unseres Systems $\sigma$ auf $C$ bestimmten Vierecke sind dem Kegelschnitte $C$ eingeschrieben und gleichzeitig dem Directionskegelschnitte $\Gamma$ umgeschrieben; sie sind demnach Poncelet'sche Polygone. Die bekannten Sätze ${ }^{1}$ ) über solche Polygone sind hier und weiter unten für den vorliegenden besonderen Fall ohneweiters evident.

Die Berühr'ungspunkte je zweier Gegenseiten eines solchen Viereckes mit $\Gamma$ ergeben sich als die Schnittpunkte der Normalen, die im Punkte $S$ auf die Verbindungsgerade derselben mit dem auf $s$ gelegenen Schnittpunkt $X$ der betreffenden Gegenseiten errichtet wird, mit den letzteren.

4. Wir wollen nun das Punktsystem $\sigma$ und seine Directionscurve $\Gamma$ ' näher untersuchen. Die Art desselben, bezw. seine Eigenschaften, ferner die Gestalt der Directionscurve $\Gamma$ und ihre Beziehungen zum Fundamentalkegelschnitt $C$ sind von der Lage des Scheitels $S$ der Strahleninvolution $J$ in Bezug auf $C$ und der Beschaffenheit von $J$ abhängig.

Zunächst ist sofort klar, dass $\sigma$ keine reellen sich selbst entsprechenden Punkte oder Coincidenzen ${ }^{2}$ ) enthalten kann, weil eine rechtwinklige Strahleninvolution elliptisch ist, also keine reellen Doppelstrahlen besitzt; daher haben $C$ und $\Gamma$ auch keine gemeinschaftlichen Tangenten.

5. Der Scheitel $S$ der Strahleninvolution $J$ liege innerhalb $C$.

Dann enthält, weil keine reellen Tangenten aus $S$ an $C$ möglich sind, $\sigma$ keine reellen Doppelpunkte ${ }^{3}$, somit auch

1) $\mathrm{Zu}$ den zahlreichen bekannten Arbeiten über Poncelet'sche Polygone kam jüngst als beachtenswert hinzu: G. Ko hn, ,Über räumliche Poncelet'sche Polygone", Sitzungsbericbte der k. Ak. d. W., Bd. CVI., 1897.

2) Nach We yr "Doppelpunkte erster Art.“

3) Nach Weyr "Doppelpunkte zweiter Art." 
keine reellen Verzweigungspunkte; daher können $C$ und ' ' in diesem Falle einander nicht schneiden. Daraus folgt weiter, dass $\Gamma$ innerhalb $C$ liegen und eine Ellipse muss sein, wenn $C$ eine Ellipse, Parabel oder spitzwinklige Hyperbel ist, hingegen eine Hyperbel, wenn $C^{\prime}$ eine stumpfwinklige Hyperbel ist. Dass bei der Hyperbel zwei Fälle zu unterscheiden sind, hat darin seinen Grund, dass auf der spitzwinkligen Hyperbel das System $\sigma$ solche Quadrupel zusammengehöriger Punkte enthalten muss, welche anf einem und demselben Curvenaste liegen, so dass die entsprechenden $\Gamma$ umgeschriebene Vierecke innerhalb des nämlichen Astes geschlossen sind, was bei einer stumpfwinkligen Hyperbel niemals der Fall sein kann; dort liegen vielmehr stets ein oder zwei Punkte auf dem andern Ast. Ein dritter möglicher Fall, die gleichseitige $\mathrm{Hy}-$ perbel betreffend, wird weiter unten im Art. 10 besprochen.

Liegt $S$ auf einer Achse von $C$, so fällt mit derselben die Hauptachse von $\Gamma$ zusammen; ihre Endpunkte werden durch die Punkte von $\sigma$ bestimmt, welche von den unter $45^{\circ}$ gegen die Achse geneigten Strahlen von $J$ auf $C$ ausgeschnitten werden. Dies gilt natürlich auch für den Fall, dass $S$ außerhalb $C$ liegt, was später nicht mehr berührt werden soll.

Fällt $S$ mit einem Brennpunkte von $C$ zusammen, dann haben $\Gamma$ und $C$ diesen Brennpunkt und die entsprechende Directrix gemeinsam und sind einander für den Brennpunkt als Centrum und die Directrix als Achse in zweifacher Art collinear.

Ist $C$ eine Ellipse und fällt $S$ mit dem Vittelpunkte derselben zusammen, so ist $s$ mit der unendlich fernen Geraden identisch. Dann wird $\Gamma$ ein mit der Ellipse concentrischer Kreis, und alle Poncelet'schen Vierecke werden Parallelogramme, sämmtlich Rhomben bis auf ein Quadrat. Sind $a$ und $b$ die Halbachsen der Ellipse $C$, so ist der Radius des Kreises $\Gamma$

$$
\rho=\frac{a b}{\sqrt{a^{2}+b^{2}}}
$$

Wenn $C$ ein Kreis und $S$ ein beliebiger innerhalb desselben gelegener Punkt ist, dann ist $\Gamma$ eine Ellipse, deren Hauptachse in den durch $S$ gehenden Durchmesser des Kreises fällt, und deren Scheitel dureh die gegen diesen Durchmesser unter $45^{\circ}$ geneigten Strahlen der Involution $J$ bestimmt sind. Ist $r$ der Radius des Kreises $C$ und $2 e$ die Entfernung seines Mittelpunktes von $S$ $(2 e<r)$, so ergibt die Rechnung für die halbe Hauptachse der Ellipse $\Gamma$ den Wert

$$
a=\frac{1}{2} \sqrt{2\left(r^{2}-2 \overline{\left.e^{2}\right)}\right.}
$$

und lehrt auch, dass der Mittelpunkt des Kreises der zweite Brennpunkt von $\Gamma$ ist.

Fallt endlich $S$ mit dem Mittelpunkte des Kreises $C$ zusammen, so ist $\Gamma$ ein mit $C$ concentrischer Kreis mit dem Radius

$$
\rho=\frac{r}{2} \sqrt{2}
$$


Die vorangehenden speciellen Fälle liefern noch eine Reihe metrischer Relationen, auf die jedoch hier nicht weiter eingegangen wird.

6. Der Scheitel $S$ der Strahleninvolution $J$ liege auberhalb $C$.

Dann wird durch $J$ ein Punktsystem $\sigma$ auf $C$ offenbar nur dann bestimmt werden können, oder es wird möglich sein, dass zwei einander entsprechende Strahlen von $J$ gleichzeitig $C$ schneiden, wenn $S$ zwischen $C$ und dem Orte $K$ der Punkte liegt, aus denen an $C$ zueinander normale Tangenten gezogen werden können, also bei der Ellipse innerhalb, bei der spitzwinkligen $\mathrm{Hy}$ perbel außerhalb des bekannten mit $C$ concentrischen Kreises $K$ und bei der Parabel zwischen Directrix und Curve. Auf der stumpfwinkligen Hyperbel hingegen, für welche $K$ imaginär ist, wird unter allen Umständen durch $J$ ein System $\sigma$ erzeugt.

In diesem Falle enthält $\sigma$ auf $C$ zwei reelle Doppelpunkte und vier reelle Verzweigungspunkte und zwar sind die ersteren die Berührungspunkte $T_{1}^{\prime}$ und $T_{2}$ der Tangenten $t_{1}$ und $t_{2}$, welche aus $S$ an $C$ gelegt werden können, und die letzteren die Schnittpunkte $V_{1}, V_{2}$ und $V_{3}, V_{4}$ der Normalstrahlen $t_{1}{ }^{\prime}$ und $t_{2}{ }^{\prime}$, welche den beiden T'angenten $t_{1}, t_{2}$ in $J$ entsprechen, mit $C$. Die Verbindungsgeraden der Doppelpunkte mit den entsprechenden Verzweigungspunkten $T_{1}$ mit $V_{1}, V_{2}$ und $T_{2}$ mit $V_{3}, V_{4}$ sind Tangenten der Directionscurve $\Gamma$ und die Verzweigungspunkte ihre Beriuhrungspunkte. Es schneiden somit $\Gamma$ und $C$ einander in vier reellen Punkten.

Mit Rücksicht auf die Entstehung und das Wesen der Doppelpunkte eines symmetrischen Punktsystems ergibt sich für unser specielles System $\sigma$, dass jeder der beiden Doppelpunkte $T_{1}$ und $T_{2}$ doppelt zu zählen ist, da durch jeden derselben zwei Tangenten von $\Gamma$ hindurchgehen. Ihre Verbindungsgerade ist die dem Brennpunkte $S$ entsprechende Directrix $s$ von $\Gamma$.

Während in dem früheren Falle, wo $S$ innerhalb $C$ liegt, jeder Punkt von $C$ ein Element des Systems $\sigma$ sein muss, ist dies hier nicht möglich, sondern es besitzt $C$ in diesem Falle parasitische Theile, deren Punkie dem System $\sigma$ nicht angehören können; dies sind offenbar diejenigen Bogen von $C$, welche innerhalb $\mathrm{F}$ liegen. Reelle Elemente von $\sigma$ enthalten nur die außerhalb $\Gamma$ gelegenen Theile von $C$; dieselben werden von zwei zusammengehörigen Verzweigungspunkten begrenzt und enthalten den nicht entsprechenden Doppelpunkt.

7. Zwischen den Tangenten von [' in den Verzweigungspunkten $V_{1}, V_{2}, V_{3}, V_{4}$ existiert eine interessante Beziehung, welche wert sein dürfte, etwas eingehender besprochen zu werden.

Diese vier Tangenten $\tau_{1}, \tau_{2}, \tau_{3}, \tau_{4}$ bilden ein vollständiges Vierseit, das die Doppelpunkte $T_{1}$ und $T_{2}$ zu Gegenecken hat, und dessen andere zwei Gegeneckenpaare $X_{1}, X_{2}$ und $Y_{1}, Y_{2}$ auf 
je einem Strahle $x$, bezw. $y$, der Involution $J$ liegen müssen. Denn ist, um für das Paar $X_{1}, X_{2}$ den Nachweis zu liefern, $G$ der Schnittpunkt des Strables $t_{1}{ }^{\prime}$ von $J$, weleher auf $C$ die Verzweigungspunkte $V_{1}, V_{2}$ ausschneidet, mit $s$, so bilden die Punkte $S, V_{1}, G, V_{2}$ eine harmonische Reihe, deren Projection aus $T_{1}$ auf den durch $X_{1}$ gehenden Strabl $x$ von $J$ die harmonische Reihe $S, X_{1}, X, X_{2}$ ist, darin $X$ der Schnittpunkt von $x$ mit $s$. In analoger Weise wird durch Projection der auf $t_{2}{ }^{\prime}$ gelegenen harmonischen Reihe $S, V_{3}, H, V_{4}$ aus $T_{2}$ auf $x$ die harmonische Reihe $S, X_{1}, X, X_{2}{ }^{\prime}$ erbalten; darin muss offenbar der Punkt $X_{2}^{\prime}$ mit dem früheren $X_{2}$, dem Schnittpunkte der Tangenten $\tau_{2}$ und $\tau_{4}$ identisch sein. Ebenso bilden auf $y$ die Punkte $S, Y_{1}, Y, Y_{2}$ eine harmonische Reihe, worin $Y$ der Schnittpunkt von $y$ mit $s$ ist.

Aus dieser Beziehung der Gegeneckenpaare $X_{1}, X_{2}$ und $Y_{1}, Y_{2}$ des oben genannten Tangentenvierseites von $\Gamma$ zum Punkte $S^{t}$ und seiner Polaren $s$ in Bezug anf $C$ folgt, dass jedem der einfachen Vierecke $X_{1} T_{1} X_{2} T_{2}^{\prime}$ und $Y_{1} T_{1} Y_{2} T_{2}$ ein Kegelschnitt $C_{1}{ }^{\prime}$, bezw. $C_{2}{ }^{\prime}$ umgeschrieben werden kann, welcher der Fundamentalkegelschnitt $C$ in den beiden Doppelpunkten $T_{1}$ und $T_{2}$ des Systems $\sigma$ berührt. $C_{1}^{\prime}$ und $C_{2}^{\prime}$ sind somit $z$ wei einander doppeltberïhrende Kegelschnitte mit $S$ und $s$ als gemeinsamem Pol and gemeinsamer Polaren.

Beachten wir, dass jede der oben genannten harmonischen Reihen auf $x$ und $y$ aus der anderen durch Projicieren aus $T_{1}$, bezw. $T_{2}$ erhalten wird, so erkennen wir $C_{1}^{\prime}$ und $C_{2}^{\prime}$ als zwei einander conjugierte ${ }^{1}$ ) oder harmonisch zugeordnete ${ }^{2}$ ) Kegelschnitte für $S$ als Centrum und $s$ als Achse der Conjunction.

Bekanntlich gibt es unendlich viele andere Kegelschnitte, deren jeder den beiden conjugierten Kegelschnitten $C_{1}^{\prime}$ und $C_{2}{ }^{7}$ gleichzeitig conjugiert ist und dieselben in denjenigen zwei Punktepaaren doppelt berührt, welche auf zwei entsprechenden Strahlen der durch $C_{1}^{\prime}$ und $C_{2}^{\prime}$ in $S$ bestimmten Strahleninvolution liegen. $\mathrm{Za}$ diesen unendlich vielen Kegelschnitten gehört insbesondere auch derjenige $C_{3}{ }^{\prime}$, welcher $C_{1}{ }^{\prime}$ in $X_{1}, X_{2}$ und $C_{2}{ }^{\prime}$ in $Y_{1}, Y_{2}$ berührt. Derselbe ist somit dem dritten in dem vollständigen Vierseit der Tangenten $\tau_{1}, \tau_{2}, \tau_{3}, \tau_{4}$ von $\Gamma$ enthaltenen einfachen Vierecke der Gegenecken $X_{1}, Y_{2}, X_{2}, Y_{1}$ umgeschrieben; seine gemeinsamen Tangentenpaare $x_{1}, x_{2}$ mit $C_{1}^{\prime}$ und $y_{1}, y_{2}$ mit $C_{2}^{\prime}$ schneiden einander in $X$, bezw. $Y$. Das Dreieck $S X Y$ ist offenbar das den drei parr'weise conjugierten Kegelschnitten gemeinsame Polardreieck ; dasselbe muss identisch sein mit dem Polardreieck, welches dem Fundamentalkegelschnitt $C$ und dem Directionskegelschnitt $\Gamma$ gemeinsam ist. Denn das gemeinsame Polardreieck von $C$ und $\Gamma$ muss als Diagonaldreieck des vollständigen Viereckes $V_{1} V_{2} V_{3} V_{4}$ der Schnittpunkte von $C$ und $\Gamma$ zusammenfallen mit dem Diagonal-

1) Wiener Chr., "Darstellende Geometrie," I. Theil, pag. $315 \mathrm{ff}$.

2) St einer-Sehröter, "Theorie der Kegelschnitte ete.," 2. Auf., pag. $383 \mathrm{ff}$. 
dreiseit des vollständigen Vierseites der Tangenten von $\Gamma$ in jenen vier Punkten, also unseres Tangentenvierseites $\tau_{1} \tau_{2} \tau_{3} \tau_{4}$; das ist aber nach dem Vorausgehenden das Dreiseit syx oder das Dreieck $S X Y$.

Diese Eigenschaft steht übrigens in innigstem Zusammenhange mit einer Beziehung zwischen dem Kegelschnitt $\Gamma$ und jedem der drei einander conjugierten $C_{1}{ }^{\prime}, C_{2}{ }^{\prime}, C_{3}{ }^{\prime}$.

8. Aus den vorangegangenen Erörterungen ist nämlich ersichtlich, dass die drei in dem vollständigen Vierseit $\tau_{1} \tau_{2} \tau_{3} \tau_{4}$ (der Tangenten von $\Gamma$ in den Verzweigungspunkten des Systems $\sigma$ )

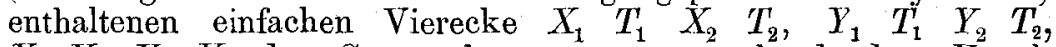

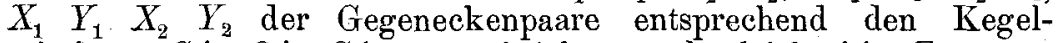
schnitten $C_{1}{ }^{\prime}, C_{2}{ }^{\prime}, C_{3}{ }^{\prime}$ eingeschrieben und gleichzeitig $\Gamma$ umgeschrieben sind; dieselben stellen somit für die Kegelschnittpaare $\Gamma C_{1}{ }^{\prime}, \Gamma C_{2}{ }^{\prime}, \Gamma C_{3}{ }^{\prime}$ Poncelet'sche Vierecke vor. $\left.{ }^{1}\right)$

Nach einem bekannten Poncelet'schen Satze sind durch diese Kegelschnittpaare weiter drei Gruppen von unendlich vielen Vierecken bestimmt, welche $\Gamma$ umgeschrieben und gleichzeitig je einem der $C_{1}{ }^{\prime}, C_{2}{ }^{\prime}, C_{3}{ }^{\prime}$ eingeschrieben sind. Unter allen Poncelet'schen Vierecken jeder solchen Gruppe ist das entsprechende von den drei oben genannten ein besonderes, nämlich von der Art, dass seine Ecken ein Quadrupel harmonischer Punkte des zugehörigen Kegelschnittes bilden. Dies erhellt aus den Erörterungen des vorigen Artikels, wonach beispielsweise das Quadrupel $T_{1} X_{1} \quad T_{2} X_{2}$ auf $C_{1}^{\prime}$ durch Projicieren der harmonischen Reihe $S X_{1} X X_{2}$ aus $T_{1}$ erhalten wird, und hat uns ja dazu geführt, $C_{1}{ }^{\prime}, C_{2}{ }^{\prime}, C_{3}{ }^{\prime}$ als drei harmonisch conjugierte Kegelschnitte zu erkennen.

9. Der Directionskegelschnitt $\Gamma$ des symmetrischen Punktsystems $\sigma$ bestimmt auf jedem der drei Kegelschnitte $C_{1}{ }^{\prime}, C_{2}{ }^{\prime}, C_{3}{ }^{\prime}$ ebenfalls ein symmetrisches Punktsystem zweiten Grades, dessen Elemente sich zu Quadrupeln grtippieren and die Ecken der entsprechenden Poncelet'schen Vierecke sind. Die Diagonalen aller einer Gruppe angehörigen Vierecke schneiden einander in einem Eckpunkte des allen Kegelschnitten gemeinsamen Polardreieckes $S X Y$, was sich sofort aus der Betrachtung des in dieser Gruppe vorkommenden Viereckes des harmonischen Quadrupels ergibt, dessen Diagonalen zwei Seiten jenes Polardreieckes sind; daraus folgt weiter, dass jedes dieser drei Systeme auch als Schnitt des bezüglichen Kegelschnittes mit einem involutorischen Strahlenbüschel

1) Jeder der drei einem der genannten Poncelet'schen Vierecke umgeschriebenen Kegelschnitte $C_{1}{ }^{\prime}, C_{2}{ }^{\prime}, C_{3}{ }^{\prime}$ ist gleichzeitig einem einfachen Vierseit $\left(x_{1} t_{1} x_{2} t_{2}, y_{1} t_{1} y_{2} t_{2}, x_{1} y_{1} x_{2} y_{2}\right)$ eingeschrieben, und zwar werden die letzteren gebildet von je zwei Gegenseitenpaaren des vollständigen Viereckes, in dessen Ecken sich je drei gemeinsame Tangenten der $C_{1}^{\prime}, C_{2}^{\prime}, C_{3}^{\prime}$ schneiden. Dieses vollständige Viereck ist dem oben wiederholt genannten vollständigen Tangentenvierseit conjugiert in Bezug auf jeden der drei Kegelschnitte, woraus eine Reihe bekannter Eigenschaften der conjugierten Kegelschnitte folgt, deren wichtigste wohl die ist, dass jeder sich selbst conjugiert ist in Bezug anf dio beiden andern. Vergl. Wiener und. Steiner-Schröter, l. c. 
(der Diagonalenpaare) erhalten wird, dessen Scheitel der entsprechende (innerhalb des Kegelschnittes gelegene) Eckpunkt des Dreieckes $S X Y$ ist.

Zwischen diesen drei neuen Systemen, ebenso zwischen ihnen und dem ursprünglichen System $\sigma$ besteht eine Reihe von Beziehungen, welche naturgemäß in dem Zusammenhange ihrer Trägerkegelschnitte untereinander und mit dem Directionskegelschnitte $\Gamma$ wurzeln. $\left.{ }^{1}\right)$ Doch wollen wir an dieser Stelle nicht weiter darauf eingehen, sondern nur noch erwähnen, dass die drei neuen Systeme keine Verzweigungspunkte, somit auch keine Doppelpunkte aufweisen, dass aber zwei derselben und zwar jene auf $C_{1}^{\prime}$ und $C_{2}{ }^{\prime}$ Coincidenzen besitzen, nämlich die Berührungspunkte der reellen gemeinschaftlichen Tangenten derselben mit $\Gamma$. Die zwei Paare von Berïhrungssehnen, welche durch $X$, bezw. $Y$, gehen müssen, stellen dann mit den zugehörigen Tangenten für $C_{1}^{\prime}$, bezw. $C_{2}^{\prime}$, je zwei degenerierte Poncelet'sche Vierecke vor, worin sowohl für den betreffenden Kegelschnitt als auch für $\Gamma$ die Berührungssehne doppelt zählt. Das dritte System, jenes auf $C_{3}{ }^{\prime}$, enthält keinerlei singuläre Elemente, ist also von ähnlicher Art wie das in Art. 5 besprochene; die dasselbe erzengende Strahleninvolution ist conlocal mit derjenigen, welche $\sigma$ auf $C$ bestimmt. Während in diesem System, bezw. für seinen Trägerkegelschnitt $C_{3}^{\prime}$ ebenso wie für den Fundamentalkegelschnitt $C$ jede beliebige Tangente von $\Gamma$ als $A n-$ fangsseite eines zu bildenden Poncelet'schen Viereckes gewählt werden kann, ist dies für $C_{1}^{\prime}$ und $C_{2}{ }^{\prime}$ nicht möglich; denn für diese Fälle besitzt $\Gamma$ zwischen den Berührungspunkten der gemeinschaftlichen Tangenten mit $C_{1}^{\prime}$ und $C_{2}^{\prime}$ parasitische Theile, deren Tangenten $C_{1}{ }^{\prime}$, bezw. $C_{2}{ }^{\prime}$, nicht in reellen Punkten schneiden.

Fassen wir nun die wichtigsten Resultate zusammen, die sich aus den Entwicklungen der letzten Artikel ergeben, so können wir den folgenden Satz aussprechen:

Die Tangenten, welche in den vier Verzweigungspunkten eines auf einem Kegelschnitte $C$ durch eine rechtwinklige Strahleninvolution ${ }^{2}$ ) mit auberhalb der Curve liegendem Scheitel erzeugten symmetrischen Punktsystems zweiten Grades anden Directionskegelschnitt $\Gamma$ desselben gezogen werden können, bilden ein vollständiges Vierseit, dessen Gegeneckenpare

1) Ist z. B. $C_{1}^{\prime}$ der mit $C$ in Bezug auf die beiden Tangenten $t_{1}$ und $t_{2}$ aus $S$ gleichartig liegende Kegelschnitt, so wird auf demselben durch die Involution $J$ ein symmetrisches Punktsystem $\sigma^{\prime}$ ausgeschnitten, das mit $\sigma$ auf $C$ die Doppelpunkte $T_{1}$ und $T_{2}$ gemeinsam hat und mit ihm für $S$ als Centrum und $s$ als Achse collinear ist. Daraus ergibt sich weiter, dass die Directionscurve $\Gamma^{\prime}$ von $\sigma^{\prime}$ collinear sein muss mit $\Gamma$, ebenso auch die hier auftretenden harmonisch conjugierten Kegelschnitte entsprechend mit den früheren u. s. w.

2) Es sei schon hier bemerkt, dass dieser Satz auch in dem allgemeinen Falle gilt, wenn die Strahleninvolution $I$ nicht rechtwinklig, sondern eine beliebige (ellipt. oder hyperb.) ist, worin die Tangenten $t_{1}, t_{2}$ von $C$ zwei beliebige Strahlen und $t_{1}^{\prime}, t_{2}^{\prime}$ die ihnen entsprechenden sind. 
$z u$ je zweien a f drei Kegelschnitten $C_{1}{ }^{\prime}, C_{2}{ }^{\prime}, C_{3}{ }^{\prime}$ liegen, vondenen zwei, $C_{1}^{\prime}, C_{2}^{\prime}$, den Fundamentalkegelschnitt $C$ in den Doppelpunkten des Punktsystems als dem einen Gegeneckenpare und den dritten, $C_{3}{ }^{\prime}$, in den beiden übrigen Gegeneckenparen des Vierseites berühren. Diesedrei Kegelschnitte $C_{1}{ }^{\prime}, C_{2}{ }^{\prime}, C_{3}{ }^{\prime}$ bilden ein Tripel (reeller) conjugierter Kegelschnitte, deren gemeinsames Polardreieck mit jenem der beiden Kegelschnitte $C$ und $\Gamma$ identisch ist; jeder von ihnen bestimmt (wie auch $C$ ) mit dem Directionskegelschnitte T eine Gruppe von unendlich vielen Poncelet'schen Vierecken, unter denendiejenigendrei, welche einem und demselben (dem oben genannten) Tangentenvierseit von $\Gamma$ angehören, Ecken besitzen, die auf dem entsprechenden der umgeschriebenen Kegelschnitte $C_{1}{ }^{\prime}, C_{2}{ }^{\prime}, C_{3}{ }^{\prime}$ je ein harmonisches Quadrupel bilden.

10. Was die Gestalt des Directionskegelschnittes $\Gamma$ in dem Falle betrifft, wo $S$ auferhalb $C$ liegt, so folgt schon aus Art. 6, dass I eine Hyperbel sein wird, wenn $C$ eine Ellipse, Parabel oder spitzwinklige Hyperbel ist, hingegen eine Ellipse, wenn $C$ eine stumpfwinklige Hyperbel ist; das letztere kann auch daraus geschlossen werden, dass in diesem Falle niemals zwei Gegenseiten eines $\Gamma$ umgeschriebenen einfachen Vierseites durch $s$ getrennt werden können.

Da die gleichseitige Hyperbel den Übergang von der spitzwinkligen zur stumpfwinkligen bildet, darf wohl angenommen werden, dass für dieselbe der Directionskegelschnitt $\Gamma$ in entsprechender Weise den Übergang von der Ellipse zur Hyperbel bilden, also eine Parabel sein wird und zwar sowohl, wenn der Punkt $S$ innerhalb als auch wenn er auferhalb. $C$ liegt.

Direct folgt dies daraus, dass die Parallelen dureh $S$ zu den Asymptoten der gleichseitigen Hyperbel zwei einander entsprechende Strahlen der Rechtwinkelinvolution $\mathcal{J}$ sind, weshalb die beiden unendlich fernen Punkte der Hyperbel zwei entsprechende Elemente von $\sigma$ bilden; daher ist die unendlich ferne Gerade als die Verbindungslinie jener zwei Punkte eine Tangente des Directionskegelschnittes $\Gamma$ und dieser somit eine Parabel.

Es ist anch ohneweiteres einleuchtend, dass die Verbindungsgerade der im Endlichen gelegenen zwei Schnittpunkte der Hyperbel mit den zu den Asymptoten parallelen Strahlen der Involution $J$ als Gegenseite der unendlich fernen Geraden im entsprechenden Poncelet'schen Viereck die Scheiteltangente der Parabel sein muss, dass ferner die Gegenseiten aller hier auftretenden Poncelet'schen Vierecke zu einander normal sind, denn ihre Schnittpunkte liegen auf $s$, der Directrix der Parabel. ${ }^{1}$ ) Das Letztere erhellt auch daraus, dass

1) Deshalb sind auch, wenn $S$ außerhalb $C$ liegt, die Verbindungsgeraden der Doppelpunkte mit den entsprechenden Verzweigungspunkten zueinander normal. 
je drei von den Punkten eines Quadrupels des aut der gleichseitigen Hyperbel liegenden Systems $\sigma$ die Ecken eines Dreieckes bilden, dessen Höhenschnittpunkt der vierte ist; in diesen Dreiecken sind die beiden einander entsprechenden Strahlen von $J$ und je zwei Gegenseiten des durcb jene bestimmten, der Hyperbel eingeschriebenen einfachen Viereckes je eine Seite und die zugehörige Höhe, ferner ist $S$ einer der drei Höhenfulopunkte.

Sind in irgend einem solchen Dreiecke neben $S$, dem Scheitel der das System $\sigma$ erzeugenden Involution $J, S^{\prime}$ und $S^{\prime \prime}$ die beiden anderen, auf $s$ gelegenen Höhenfufpunkte, so kann jeder derselben als der Scheitel einer rechtwinkligen Strahleninvolution $J^{\prime}$, bezw. $J^{\prime \prime}$, betrachtet werden, welche auf $C$ ein symmetrisches Punktsystem zweiten Grades ' $\sigma$ ', bezw. $\sigma$ ', erzeugt, welche beide mit dem ursprünglichen System $\sigma$ ein Quadrupel, nämlich das von den Ecken und dem Höhenschnittpunkte jenes Dreieckes gebildete, gemeinsam haben. Daraus ergibt sich eine interessante Beziehung zwischen den entsprechenden drei Parabeln, womit wir uns an anderem Orte eingehender befassen wollen.

Aus den weiter oben stehenden Erörterungen dieses Artikels folgt noch ein (zum Theil in anderer Form Bekanntes enthaltender) Satz iiber Dreiecke, welche einer gleichseitigen Hyperbel eingeschrieben sind:

Werden einer gleichseitigen Hyperbel Dreiecke eingeschrieben, die einen nicht auf der Curve gelegenen Punkt $S$ als gemeinsamen Höhenfußpunkt besitzen, so liegen die Paare der anderen Höhenfuljpunkte $S^{\prime}, S^{\prime \prime}$ alle anf einer Geraden $s$, welche die Polare des gemeinsamen Höhenfubpunktes in Bezug auf die Hyperbel ist. Die nicht durch $S$ gehenden Seiten und Höhen aller jener Dreiecke hüllen eine Parabel ein, welche $S$ zum Brennpunkte and $s$ zur Directrix hat.

11. In dem Grenzfalle; wo der Scheitel $S$ der Rechtwinkelinvolution $J$ auf dem Orte $K$ der Punkte liegt, aus denen an $C$ zwei zueinander normale Tangenten gezogen werden können, reduciert sich $\sigma$ auf die beiden Berührungspunkte derselben, in denen Doppelpunkte und Verzweigungspunkte zusammenfallen, und $\Gamma$ auf die doppelt zu zählende Polare $s$ von $S$.

Der Fall endlich, wo $S$ a uf $C$ selbst liegt, führt zu keinem Punktsystem $\sigma$, sondern nur zu einer Involution $i$ auf $C$. Wenn wir ihm dennoch an dieser Stelle einige Aufmerksamkeit schenken, so sei dies mit Rücksicht auf die große Einfachheit gestattet, mit der aus ihm etliche Eigenschaften der gleichseitigen Hyperbel abgeleitet werden können, von denen manche weniger oder nicht bekannt sein dürften, während andere sonst auf einem von diesem verschiedenen Wege erhalten werden. ${ }^{1}$ )

1) Vergl, bezüglich mehrerer Eigenschaften der gleichseitigen Hyperbel auch E. Czuber, "Über die einem Kegelschnitte umgeschriebenen Kreisvierecke ${ }^{*}$ im Archiv für Math. n. Phys., II. R., 9. Thl., pag. 103 ff. 
Fine rechtwinklige Strahleninvolution $J$, deren Scheitel $S$ auf einem Kegelschnitt $C$ liegt, schneidet auf diesem eine Involution $i$ aus, deren Centrum $N$ bekanntlich ${ }^{1}$ ) der Schnittpunkt der Normalen $n$ von $C$ im Punkte $S$ mit demjenigen Durchmesser $d_{n}$ ist, der zu dem durch $S$ gehenden $d_{s}$ in Bezug auf die Achsen symmetrisch liegt. Hiebei können wir auch die Verbindungsgeraden entsprechender Punkte der Involution $i$ als diejenigen Sehnen von $C$ betrachten, welche von $S$ aus unter rechtem Winkel erscheinen, oder als die Hypotenusen derjenigen rechtwinkligen Dreiecke, welche $C$ eingeschrieben sind und in $S$ den Scheitel des rechten Winkels haben.

Ist nun $C$ eine gleichseitige Hyperbel, so fällt für jede beliebige Wahl des Punktes $S$ das Centrum $N$ der Involution $i$ mit dem unendlich fernen Punkte der Normale $n$ zusammen, weil das der Hyperbel eingeschriebene rechtwinklige Dreieck, dessen Katheten zu den Asymptoten parallel sind, die unendlich ferne Gerade zur Hypotenuse hat; das Büschel $N$ der Sehnen, welche entsprechende Punkte von $i$ verbinden, ist somit in diesem Falle ein Parallelstrahlenbüschel. Daraus ergibt sich der Satz:

Alle Sehnen einer gleichseitigen Hyperbel, welche von irgend einem ihrer Punkte unter rechtem Winkel erscheinen, sind parallel zur Normalen in diesem Punkte.

Da die Normalen einer Hyperbel nur zu reellen Durchmessern parallel sein können, sind unter den parallelen Sehnen auch nur solche ron diesen Richtungen verstanden. Unter jhnen befindet sich auch ein Durchmesser $d_{n}$, der zu dem durch $S$ gehenden $d_{s}$ in Bezug auf die Achsen symmetrisch liegt; daraus folgt:

Normale und Durchmesser für einen beliebigen Punkt der gleichseitigen Hyperbel sind gegen die Achsen unter gleichen aber entgegengesetzt bezeichneten Winkeln geneigt; und daraus weiter:

Die Subnormale für jeden Punkt der gleichseitigen Hyperbel ist gleich der Abscisse.

Aus der durch die Parallelverschiebung der Sehnen hervorgerufenen Drehung des rechten Winkels um $S$ ist zu erkennen:

Aus einem Punkte einer gleichseitigen Hyperbol erscheinen solche Bogen derselben, welche zwischen beliebigen zwei, zur Normalen jenes Punktes parallelen Sehnen liegen, unter gleichen Winkeln.

Da die Normale $n^{\prime}$ in $S^{\prime}$, dem zweiten Endpunkte des durch $S$ gehenden Durchmessers $d_{s}$, parallel ist zu jener $n$ in $S$, so werden offenbar alle oben genannten parallelen Sehnen auch von $S^{\prime}$ aus unter rechtem Winkel gesehen. Wir können somit sagen:

Der über einer (zu einem reeilen Durchmesser parallelen) Sehne als Durchmesser beschriebene Kreis schneidet die gleichseitige Hyperbel in zwei Punkten, in denen die Normalen parallel sind zu

1) Poncelet, "Traité des propriétés etc.", pag. 280. Steiner, "Gesammelte Werke", 2. Bd, pag. 432. 
jener Sehne. Diese beiden Punkte sind die Endpunkte desjenigen Durchmessers, der gegen die Achsen unter gleichen aber entgegengesetzt bezeichneten Winkeln geneigt ist wie die Sehne; es sind somit die Halbierungsgeraden der von Sehne und Durchmesser gebildeten Winkel der Achsenrichtungen.

Alle Kreise, welche durch die Endpunkte $S, S^{\prime}$ eines Durchmessers der gleichseitigen Hyperbel gehen, bilden ein Büschel, dessen Centrale conjugiert ist der Richtung der zu den Normalen der Curve in $S$ und $S^{\prime}$ parallelen Chordalen, welche die Kreise des Büschels mit der Hyperbel bestimmen.

Daraus ergibt sich weiter durch Umkehrung:

Die Kreise eines Büschels werden von ihren zu irgend einer Richtung parallelen Durchmessern in Punkten einer gleichseitigen Hyperbel geschnitten, für welche die beiden Scheitel $S$ und $S^{\prime}$ des Kreisbüschels Endpunkte eines Durchmessers $d_{s}$ sind. Die Halbierungsgeraden der Winkel, welche der dem kleinsten Kreise angehörige $d_{n}$ jener Durchmesser mit der Chordale des Büschels bildet, sind die Achsen, jene der Winkel, welche der genannte Durchmesser mit der Centrale des Büschels bildet, die Asymptoten der Hyperbel. ${ }^{1}$ )

Die voranstehenden Sätze können noch weiter specialisiert werden, indem man die Punkte $S$ und $S^{\prime}$ in die beiden Scheitel der Hyperbel verlegt; auf diese Weise erhält man Folgendes:

Älle Sehnen einer gleichseitigen Hyperbel, welche von den Scheiteln aus unter rechtem Winkel erscheinen, sind parallel zur Hauptachse. ${ }^{2}$ )

Die zwischen zwei zur Hauptachse parallelen Sehnen liegenden Bogen einer gleichseitigen Hyperbel werden von ihren Scheiteln aus unter gleichen Winkeln gesehen u. a. m.

Dass einige der obenstehenden Sätze ohneweiters zur Construction der gleichseitigen Hyperbel, ebenso auch zur Construction von Normalen und Tangenten benützt werden können, ist augenscheinlich und bedarf keiner weiteren Erläuterung.

12. Nach den Erörterungen des Art. 1 ist der Directionskegelschnitt $\Gamma$ unseres auf $C$ liegenden Punktsystems $\sigma$ durch irgend ein Quadrupel $A_{1}, A_{2} ; A_{1}{ }^{\prime}, A_{2}^{\prime}$ zusammengehöriger Punkte vollkommen bestimmt, da noch $S$ als Brennpunkt hinzutritt. Legen wir durch diese vier Punkte einen beliebigen anderen Kegelschnitt $C$, so ist klar, dass das durch die rechtwinklige Strahleninvolution $J$ auf $C^{\prime}$ erzeugte Punktsystem $\sigma^{\prime}$ denselben Directionskegelschnitt $\Gamma$ wie $\sigma$ auf $C$ besitzen muss, da derselbe bereits durch das auch auf $C^{\prime \prime}$ liegende Quadrupel $A_{1}, A_{2} ; A_{1}{ }^{\prime}, A_{2}{ }^{\prime}$ bestimmt ist.

1) Vergl. N. Czuber, l. c., pag. 107.

2) Vergl diesbezüglich W. Fied ler, „Darstell. Geometrie", 3. Aufl, 1. Thl, pag. 197, wo die gleichseitige Hyperbel durch involutorische Collineation aus dem Kreise mit einem Punkte desselben als Centrum und dem anf dem zugehörigen Radius normalen Durchmesser als Gegenachse seines Systems erhalten wird. 
Mit Rücksicht darauf, dass durch ein solches Quadrupel ein Kegelschnittbüschel festgelegt ist, erhalten wir somit den tolgenden Satz:

Eine rechtwinklige Strahleninvolution erzeugt auf allen Kegelschnitten eines Büschels, dessen Scheitel vier auf $z$ wei entsprechenden Strablen der Involution parweise gelegene Punkte sind, symmetrischePunktsystemezweiten Grades, welcheeinen gemeinsamen Directionskegelschnitt besitzen; derselbe ist dem durch die vier Scheitel des Büschels bestimmten einfachen Vierecke eingeschrieben, worin keine zwei a ufeinanderfolgende Ecken a f einem Strahle jener Involution liegen, und hat den Schnittpunkt der Diagonalen desselben zum Brennpunkte.

Ein Kegelschnittbüschel, dessen sämmtliche Scheitel reell sind und eine beliebige gegenseitige Lage besitzen, enthält bekanntlich stets eine gleichseitige Hyperbel. Diese ist in dem vorliegenden Falle keine eigentliche Hyperbel, sondern eine degenerierte und besteht aus den beiden aufeinander normalen Diagonalen des vorhin genannten einfachen Viereckes der Scheitel; $\Gamma$ ist hiebei eine Ellipse oder Hyperbel. Sind jedoch die Scheitel so gelegen, dass jeder der Höhensehnittpunkt des von den drei übrigen gebildeten Dreieckes ist, so sind bekanntlich alle Kegelschnitte des betreffenden Büschels gleichseitige Hyperbeln, und $\Gamma$ ist dann, was uns schon der vorige Artikel gelehrt hat, eine Parabel.

Zu den Kegelschnitten des durch irgend ein Quadrupel von $\sigma$ bestimmten Büschels gehören ferner noch die zwei degenerierten, welche aus den Gegenseitenparen des oben bezeichneten einfachen Viereckes bestehen. Auf jedem derselben erzeugt die Rechtwinkelinvolution $J$ zwei involutorische Punktreihen; die Verbindungsgeraden entsprechender, d.h. auf zwei zueinander normalen Strahlen von $J$ gelegener, Punkte derselben hüllen einen Kegelschnitt ein, der offenbar wieder $\Gamma$ ist. ${ }^{1}$ ) Jeder dieser zwei degenerierten Kegelschnitte des Büschels kann zu einer einfachen Ermittlung des zweiten Brennpunktes und der Achsen von $\Gamma$ benützt werden. Ist nämlich $X$ der Schnittpunkt zweier Gegenseiten $\gamma_{1}$ und $\gamma_{2}$ des Viereckes, so sind nach Früherem die Berührungspunkte $\Delta_{1}$ und $\Delta_{2}$ derselben mit $\Gamma$ ihre Schnittpunkte mit der Normalen, die in $S$ auf $S X$ errichtet wird; sind ferner $G_{1}$ und $G_{2}$ die Gegenpunkte des Brennpunktes $S$ in Bezug auf $\gamma_{1}$ und $\gamma_{2}$, so sehneiden einander die Geraden $G_{1} \Delta_{1}$ und $G_{2} \Delta_{2}$ im zweiten Brennpunkte $F$, und $F G_{1}$, sowie $F G_{2}$ ist die Länge der Hauptachse.

1) Dies führt zu dem bekannten Satze, wonach die von zwei festen 'I'angenten eines Kegelschnittes auf einer beweglichen 'Tangente begrenzte Strecke von einem Brennpunkte ans unter constantem Winkel erscheint, der in diesem Falle, wo der Schnittpunkt der beiden festen Tangenten auf der dem Brennpunkt entsprechenden Directrix liegt, ein Rechter ist. 
Wenn wir in den beiden Punktinvolutionen, von denen irgend zwei einander entsprechende Strahlen der Rechtwinkelinvolution $J$ von allen Kegelschnitten eines durch ein Quadrupel zusammengehöriger Punkte des Systems $\sigma$ bestimmten Büschels geschnitten werden, diejenigen Paare einander als entsprechend zuordnen, welche auf demselben Kegelschnitt liegen, so erhalten wir auf jenen zwei Strahlen auch ein symmetrisches Punktsystem zweiten Grades $^{1}$ ), dessen Directionscurve ebenfalls $\Gamma$ ist. Diese $z$ usammengehörigen Punktepaare der beiden Involu tionen sind aber die Gegeneckenpare derjenigen durch $\Gamma$ und die einzelnen Kegelschnittedes Büschels bestimmten Poncelet'schen Vierecke, deren Diagonalen in dieselben zwei zueinander normalen Geraden fallen.

Am Schlusse dieser Betrachtung sei noch kurz darauf hingewiesen, dass es, nachdem durch jedes der auf $C$ gelegenen Quadrupel von $\sigma$ ein Kegelschnittbiischel festgelegt wird, unendlich viele solcher Büschel gibt, deren jedes mit der Rechtwinkelinvolution $J$ unendlich viele symmetrische Punktsysteme zweiten Grades bestimmt, welche sämmtlich $\Gamma$ als gemeinschaftlichen Directionskegelschnitt besitzen.

Die Anzahl der unendlich vielen Gruppen von je unendlich vielen Poncelet'schen Vierecken, welche durch die einzelnen Kegelschnitte eines und desselben Büschels mit $\Gamma$ bestimmt werden, wird aber durch jene unendlich vielen Büschel nicht vermehrt.

Dies kann schon aus der Mannigfaltigkeit des Punktes in der Ebene geschlossen werden, ist aber auch einleuchtend, wenn man die sämmtlichen hier auftretenden Vierecke so ordnet, dass alle diejenigen (unendlich vielen), deren Diagonalen in zwei entsprechende Strahlen von $J$ fallen, zu einer Gruppe vareinigt werden, wie dies oben geschehen ist; solche Gruppen gibt es mit den Strahlenpaaren von $J$ unendlich viele.

Jedes der hier in Betracht kommenden $\Gamma$ umgeschriebenen Vierecke muss einer solchen Gruppe angehören; denn ist $A_{1}$ irgend ein außerhalb $\Gamma$ gelegener Punkt der Ebene, welcher als Ausgangspunkt zur Bildung eines solchen Viereckes gewählt wird, $a$ der Strahl, der ihn mit $S$ verbindet, und $a^{\prime}$ der darauf normale Strahl von $J$, so schneiden die beiden Tangenten aus $A_{1}$ von $\Gamma$ auf $a^{\prime}$ die Punkte $A_{1}{ }^{\prime}$ und $A_{2}{ }^{\prime}$ aus, und die zweiten Tangenten aus diesen Punkten an $\Gamma$ schneiden einander auf $a$ im vierten Eckpunkte $A_{2}$ des Viereckes.

13. Die Strahleninvolution $J$ sei eine beliebig elliptische.

Dann sind die Eigensehaften der bei verschiedenen Lagen des Scheitels $S$ durch $J$ auf $C$ bestimmten symmetrischen Punktsysteme $\sigma$ von jenen der bisher betrachteten, welche durch eine Rechtwinkelinvolution erzeugt wurden, wenig verschieden.

1) Oder zwei projectivische Involutionen. 
Da die elliptische aus der rechtwinkligen Strahleninvolution durch Projicieren erhalten werden kann, so gelten diejenigen der früheren Sätze, welche nur von projectivischen Eigenschaften handeln, unverändert auch hier im allgemeineren Falle, so insbesondere jener in Art. 9 über das Tripel conjugierter Kegelschnitte für $C$ als Fundamentalkegelschnitt. Andere erfordern nur eine geringfügige Umänderung; so wird hier der Directionskegelschnitt $\Gamma^{\prime}$ eine Parabel werden, wenn $C$ eine Hyperbel ist, deren Asymptoten zu zwei entsprechenden Strahlen von $J$ parallel sind.

Ein wesentlicher Unterschied gegen früher besteht offenbar darin, dass $S$ und $s$ wohl dem gemeinsamen Polardreieck von $C$ und $\Gamma$ als Ecke und Gegenseite angehören, jedoch nicht Brennpunkt und entprechende Directrix von $\Gamma$ sind. Dies ist die Ursache der vorhin erwähnten Veränderung gewisser früheren Sätze; darauf näher einzugehen ist wohl nicht nöthig.

Nur der specielle Fall soll hier berührt werden, der eintritt, wenn der Scheitel $S$ der elliptischen Strahleninvolution $J$ mit dem Mittelpunkte von $C$ zusammenfällt.

Dann ist jedes durch ein Quadrupel zusammengehöriger Punkte von $\sigma$ bestimmte Poncelet'sche Viereck, das $Q$ ein- und $\Gamma$ umgeschrieben ist, ein Parallelogramm; die Diagonalen desselben sind zwei einander entsprechende Strahlen von $\vec{J}$ und bilden ein Paar conjugierter Durchmesser des Directionskegelschnittes $\Gamma$. Derselbe ist offenbar eine Ellipse, deren Achsen mit den Normalstrahlen der Involution $J$ zusammenfallen.

Da die Directionskegelschnitte $\Gamma$ aller auf (unter obiger Voraussetzung) beliebig gewählten Kegelschnitten $C$ erzeugten Punktsysteme $\sigma$ dieselbe Involution conjugierter Durchmesser, nämlich $J$, besitzen, können wir sagen:

Eine elliptischeStrahleninvolution schneidet auf allen mit ihr concentrischen Kegelschnitten symmetrische Punktsysteme zweiten Grades aus, deren Directionskegelschnitte coachsiale und ähnliche Ellipsen sind.

Wird schlieblich - als speciellster Fall - $C$ ein Kreis, so gehen die $C$ ein- und $\Gamma$ umgeschriebenen Parallelogramme in Rechtecke über und der Directionskegelschnitt $\Gamma$ in jene Ellipse, für welche der Kreis $C^{\prime}$ der Ort der Punkte mit zueinander normalen Tangenten ist. ${ }^{1}$ )

2) Dio Achsenlängen dieser Ellipse $I$ sind durch dasjenige $C$ ein- und $\Gamma$ umgesehriebene Rechteck bestimmt, dessen Seiten zu den Achsen von $I$ parallel sind; die Diagonalen desselben sind demnach die den kleinsten Winkel miteinander einschließenden Strahlen von $J$, fallen also mit den za den Achsen symmetrisch gelegenen conjugierten Durchmessern von $I$ zusammen.

Die Ermittlung jener zwei Strahlen von $J$ lässt sich mit der Bestimmung der Normalstrahlen zum kürzesten Verfahren vereinigen, indem man in einem beliebigen durch den Scheitel $S$ von $J$ gelegten Kreis, auf dem $J$ eine Involution $i$ mit dem Centrum $N$ ausschneidet, die Endpunkte des durch $N$ gehenden Durchmessers und der zu demselben normalen durch $N$ gehenden Selune mit $S$ verbindet. 


\section{bolische.}

14. Die Strahleninvolution $J$ sei eine hyper-

Da je zwei entsprechende Strahlen ron $J$ durch die Doppelstrahlen $d_{1}$ und $d_{2}$ getrennt werden, so ist klar, dass eine hyperbolische Strahleninvolution $J$ auf einem Kegelschnitt $C$ nur dann ein reelles Punktsystem $\sigma$ erzeugen kann, wenn mindestens einer der beiden Doppelstrahlen von $C$ geschnitten wird; in diesen Schnittpunkten fallen offenbar je zwei einander entsprechende Punkte von $\sigma$ zusammen, sie sind also sich selbst entsprechende oder Coincidenzpunkte von $\sigma$. Es ist wohl ohneweiters einleuchtend, dass die Tangenten von $C$ in diesen Punkten auch Tangenten des Directionskegelschnittes $\Gamma$ sein müssen.

Im Folgenden sollen nun die hauptsächlichsten der aus den verschiedenen Lagen des Fundamentalkegelschnittes $C$ gegen $J$ resultierenden Fälle kurz besprochen werden.

15. Der Scheitel $S$ von $J$ liege innerhalb $C$.

In diesem Falle werden beide Doppelstrahlen $d_{1}$ and $d_{2}$ der Involution $J$ von $C$ in den Punktepaaren $D_{1} D_{1}^{\prime}$ und $D_{2} D_{2}^{\prime}$ geschnitten, so dass also sämmtliche der vier möglichen Coincidenzpunkte von $\sigma$ reell sind. Hingegen treten, da kein Strahl von $J$ Tangente von $C$ sein kann, in $\sigma$ keine reellen Doppelpunkte und somit auch keine reellen Verzweigungspunkte auf; daher können $C$ und $\mathrm{T}$ einander nicht schneiden.

$\mathrm{Zu}$ den ausgezeichneten Tangenten des Directionskegelschnittes $\Gamma$ gehören nebst den Tangenten $\delta_{1}, \delta_{1}^{\prime}, \delta_{2}, \delta_{2}^{\prime}$ von $C$ in den Coincidenzpunkten $D_{1}, D_{1}^{\prime}, D_{2}, D_{2}^{\prime}$ auch noch die Doppelstrahlen $d_{1}$ und $d_{2}$ von $J$, was aus Folgendem erhellt.

Ein durch irgend ein Quadrupel zusammengehöriger Punkte von $\sigma$ bestimmtes $C$ ein- und $\Gamma$ ungeschriebenes Viereek degeneriert, wenn zwei einander entsprechende (nicht auf demselben Strahl von $J$ gelegene) Punkte sich in einem Coincidenzpunkte vereinigen, in den als Tangente von $\Gamma$ offenbar doppelt zu zählenden entsprechenden Doppelstrahl und die Tangenten von $C$ in den dazu gehörigen zwei Coincidenzpunkten.

Es liefern somit im vorliegenden Falle (und auch in dem späteren, wo $S$ außerhalb $C$ liegt und beide Doppelstrahlen geschnitten werden) zwei degenerierte Poncelet'sche Vierecke die zwei Tangententripel $d_{1}, \delta_{1}, \delta_{1}^{\prime}$ und $d_{2}, \delta_{2}, \delta_{2}^{\prime}$, welche sich zu einem Brianchon'schen Sechsseit vereinigen, durch das $\mathrm{F}$ mehr als hinreichend bestimmt ist.

Da $S$ and $s$ wieder wie früher Ecke und Gegenseite des $C$ und $\Gamma$ gemeinsamen Polardreieckes bilden, so sind die Schnittpunkte $I$ und $I I$ von $s$ mit den Doppelstrahlen $d_{1}$ und $d_{2}$ die Berührungspunkte der letzteren mit dem Directionskegelschnitte $\Gamma$. Die gemeinsame Polare $s$ von $S$ in Bezug auf $C$ und $F$ ist offenbar bereits durch das Viereck der Coincidenzpunkte bestimmt, und es 
bildet $S$ mit jedem der beiden Berïhrungspunkte $I$ und $I I$ auf $\Gamma$ und den auf dem entsprechenden Doppelstrahl gelegenen zwei Coincidenzpunkten je eine harmonische Reihe.

$C$ und $\Gamma$ bestimmen eine Kegelschnittschar mit den reellen Grundtangenten $\delta_{1}, \delta_{1}^{\prime}, \delta_{2}, \delta_{2}^{\prime}$; daraus und aus dem Zusammenhange dieser zwei besonderen Kegelschnitte der Schar ergeben sich einige interessante Beziehungen, auf die jedoch hier nicht weiter eingegangen sondern bloß hingewiesen werden soll. ${ }^{1}$ )

Wenn $C$ eine Hyperbel ist, deren Asymptoten zu zwei einander entsprechenden Strablen von $J$ parallel sind, fallen zwei Punkte des durch dieses Strahlenpaar erzeugten Quadrupels von $\sigma$ mit den unendlich fernen Punkten von $C$ zusammen; die unendlich ferne Gerade ist somit eine Tangente des Directionskegelschnittes $\Gamma$ und dieser selbst eine Parabel.

Sind die Asymptoten der Hyperbel $C$ zu den Doppelstrahlen $d_{1}$ und $d_{2}$ von $J$ parallel, dann sind zwei Coincidenzpunkte von $\sigma$ mit den unendlich fernen Punkten von $C$ identisch, so dass die Asymptoten von $C$ Tangenten von $\Gamma$ sind. Die gemeinsame Polare $s$ von $S$ ist parallel zur Verbindungsgeraden der beiden im Endlichen gelegenen Coincidenzpunkte, und diese selbst halbieren die Strecken zwischen $S$ und den Berührungspunkten $I$, bezw. II, der Doppelstrahlen $d_{1}$ und $d_{2}$ mit $\Gamma$, denn die ihnen in Bezug auf $S$ und $I$, bezw. $S$ und $I I$, conjugierten Punkte liegen im Unendlichen.

Fällt der Scheitel $S$ der hyperbolischen Strahleninvolution $J$ mit dem Mittelpunkte von $C$ zusammen, wobei es gleichgiltig bleibt, ob $C$ eine Ellipse oder Hyperbel ist, ob also $S$ innerhalb oder außerhalb $C$ liegt, so erhalten wir in analoger Weise wie in dem Falle, wo $\mathcal{J}$ elliptisch ist, als Poncelet'sche Vierecke Parallelogramme, deren Diagonalen je zwei entsprechende Strahlen von $J$ sind und conjugierte Durchmesser von $\mathrm{F}$ bilden. $\mathrm{l}$ " selbst ist eine Hyperbel, deren Acbsen mit den Normalstrahlen von $J$ zusammenfallen.

$\mathrm{Da}$ in diesem Falle $s$ mit der unendlich fernen Geraden zasammenfällt, die Berührungspunkte $I$ und $I I$ der Doppelstrahlen $d_{1}$ und $d_{2}$ von $J$ mit $\Gamma$ somit die unendlich fernen Punkte dieser Geraden sind, so bilden die Doppelstrablen von $J$ die Asymptoten der Directionskegelschnitte aller auf diese Weise erzeugten symmetrischen Punktsysteme, so dass wir sagen können:

Eine hyperbolische Strahleninvolution schneidet auf allen mit ihr concentrischen Kegelschnitten

1) So bilden beispielsweise die Tangenten von $C$ in den vier Coincidenzpunkten mit jedem der drei Gegenseitenpare des durch dieselben bestiminten voll. ständigen Viereckes je ein Brianchon'sches Sechsseit, worin die Schnittpunkte des betreffenden Paares von Gegenseiten mit der nicht durch ihren Schnittpunkt gehenden Seite des Diagonaldreieckes die Berührungspunkte des zugehörigen Kegelschnittes mit jenen Seiten sind.

Jeder Diagonaleckpunkt des vollständigen Viereckes der Coincidenzpunkte ist der Scheitel einer hyperbolischen Strahleninvolution, welche die in diesem Diagonalpunkte sich schneidenden Gegenseiten des Viereckes zu Doppelstrahlen hat und auf $C$ ein symmetrisches Punktsystem zweiten Grades orzeugt, das den entsprechenden der genannten drei Kegelschnitte als Directionskegelschnitt besitzt. 
symmetrische Punktsysteme zweiten Grades aus, deren Directionskegelschnitte coachsiale und ähnliche Hyperbeln sind.

Ist endlich $C$ ein Kreis, dessen Mittelpunkt mit $S$ zusammenfällt, so gehen die durch die Quadrupel zusammengehöriger Punkte von $\sigma$ bestimmten Poncelet'schen Vierecke in Rechtecke und der Directionskegelschnitt $\Gamma$ in diejenige Hyperbel über, für welche die Doppelstrahlen der Involution $J$ die Asymptoten bilden und der Kreis $C$ der Ort der Punkte mit zueinander normalen Tangenten ist.

16. Der Scheitel $S$ von $J$ liege auberhalb $C$.

Setzen wir zunächst voraus, dass $C$ von beiden Doppelstrahlen $d_{1}$ und $d_{2}$ der Involution $J$ geschnitten werde, so haben wir den Fall vor uns, dass alle singulären Elemente des Punktsystems $\sigma$ reell sind: vier Coincidenzpunkte $D_{1}, D_{1}^{\prime} ; D_{2}, D_{2}^{\prime}$ (die Schnittpunkte der Doppelstrahlen $d_{1}, d_{2}$ mit $C$ ), zwei Doppelpunkte $T_{1}$. $T_{2}$ (die. Berührungspunkte der Tangenten $t_{1}, t_{2}$ aus $S$ an $C$ ) und vier Verzweigungspunkte $V_{1}, V_{2} ; V_{3}, V_{4}$ (die Schnittpunkte der den Tangenten $t_{1}, t_{2}$ in der Involution $J$ entsprechenden Strahlen $t_{1}^{\prime}, t_{2}^{\prime}$ mit $\left.C^{\prime}\right)$; hiebei sind die Doppelpunkte $T_{1}, T_{2}$ doppelt zu zählen, da sich in jedem die Tangenten von $\Gamma$ in den zwei auf demselben Strahl von $J$ gelegenen Verzweigungspunkten treffen.

Infolge der reellen Verzweigungspunkte schneiden hier $C$ und $\Gamma$ einander in vier reellen Punkten.

Für die Bestimmung des Directionskegelschnittes $\Gamma$ liefern die singulären Elemente von $\sigma$ schon überzählige Stücke und zwar die Verzweigungspunkte und deren Verbindungsgeraden mit den entsprechenden Doppelpunkten vier Punkte sammt den dazu gehörigen Tangenten und die Tangenten von $C$ in den Coincidenzpunkten vier weitere Tangenten; außerdem sind noch die beiden Doppelstrahlen von $J$ Tangenten von $\Gamma$ und ihre Schnittpunkte mit der Polaren $s$ von $S$ die Berührungspunkte derselben.

Beachtenswert ist, dass das $C$ und $\Gamma$ gemeinsam umschriebene Vierseit der Tangenten $\delta_{1} \delta_{1}^{\prime} \delta_{2} \delta_{2}^{\prime}$ von $C$ in den Coincidenzpunkten und das $\Gamma$ umschriebene Vierseit der Tangenten $\tau_{1} \tau_{2} \tau_{3} \tau_{4}$ von $\Gamma$ in den Verzweigungspunkten ein gemeinsames Diagonaldreiseit oder die vollständigen Vierecke der Coincidenzpunkte $D_{1} D_{1}^{\prime} D_{2} D_{2}^{\prime}$ und der Verzweigungspunkte $V_{1} V_{2} V_{3} V_{4}$ ein gemeinsames Diagonaldreieck besitzen.

In der That, ist $S X X^{\prime}$ das Diagonaldreieck des Viereckes $D_{1} D_{1}^{\prime} D_{2} D_{2}^{\prime}$, so ist auf Grund einer Figenschaft der quadratischen Involutionen leicht einzusehen, dass die nicht durch $S$ gehenden Paare von Gegenseiten des Viereckes $V_{1} V_{2} V_{3} V_{4}$ sich in $X$ und $X^{\prime}$ auf $s$ schneiden müssen. 
Da nämlich $t_{1}, t_{1}^{\prime}$ und $t_{2}, t_{2}^{\prime}$ in der Strahleninvolution $J$ einander entsprechende Strahlen und $d_{1}, d_{2}$ die Doppelstrahlen sind, so gehören bekanntlich auch die Strahlenpaare $t_{1}, t_{2} ; t_{1}^{\prime}, t_{2}^{\prime} ; d_{1}, d_{2}$ einer anderen Involution an. Die Doppelstrahlen dieser letzteren, sind offenbar die durch $S$ gehenden Seiten $x$ und $x^{\prime}$ des Diagonaldreieckes $S X X^{\prime}$, denn sie trennen als conjugierte Geraden in Bezug auf $C \quad t_{1}$ und $t_{2}$ und als durch $S$ gehende Seiten des Diagonaldreieckes die durch denselben Punkt gehenden Seiten $d_{1}$ und $d_{2}$ des Viereckes $D_{1} D_{1}^{\prime} D_{2} D_{2}^{\prime}$ harmonisch, und somit als Doppelstrahlen von $J^{r}$ auch in gleicher Weise das dritte Strahlenpaar $t_{1}^{\prime}, t_{2}^{\prime}$, von $J^{\prime}$, welche die durch $S$ gehenden Gegenseiten des Viereckes $V_{1} V_{2} V_{3} V_{4}$ sind.

Aus dieser Betrachtung erhellt auch, dass $x$ und $x^{\prime}$ zwei entsprechende Strahlen der Involution $J$ sind.

Auf die Bedeutung der Diagonalecken $X$ und $X^{\prime}$ und der sich in ihnen schneidenden Gegenseitenpaare der Vierecke kommen wir am Schlusse nochmals zu sprechen; es soll hier nur bemerkt werden, dass $X, X^{\prime}$ und $S^{\prime}$ als Brianchon'sche Punkte in aus den zehn wiederholt genannten Tangenten von $\Gamma$ gebildeten Brianchon'schen Sechsseiten auftreten, und dass durch sie insbesondere die Geraden hindurchgehen, aut denen parweise die Berührungspunkte gleichartiger (derselben Gruppe angehöriger) Tangenten mit $C$ und mit $F$ liegen.

Die Erörterungen der Artikel 7,8 und 9 und der Schlusssatz über das Tripel conjugierter Kegelschnitte, welches durch das Tangentenvierseit $\tau_{1} \tau_{2} \tau_{3} \tau_{4}$ bestimmt ist, gelten in vollem Umfange auch für den vorliegenden Fall der hyperbolischen Involution.

17. In dem besonderen Falle, wo nur einer der beiden Doppelstrahlen der Involution $J$ den Kegelschnitt $C$ schneidet, enthält das entsprechende Punktsystem $\sigma$ nur je zwei reelle Coincidenz- und Verzweigungspunkte, während die andern zwei imaginär sind; hingegen sind wie früher beide Doppelpunkte und die Berührungspunkte beider Doppelstrahlen mit $\Gamma$ reell. Die beiden reellen Verzweigungspunkte zerlegen $C$ in zwei Theile, wovon derjenige, auf dem der diesen Verzweigungspunkten entsprechende Doppelpunkt liegt, durchwegs Quadrupel reeller Punkte von $\sigma$ enthält, die also je vier Tangenten von $\Gamma$ liefern, während der andere Theil parasitisch ist; derselbe liefert, da ein Punktepaar oder beide eines jeden Quadrupels imaginär sind, keine Tangenten von $\Gamma$, er liegt innerhalb $\Gamma$.

Wenn $C$ von dem einen Doppelstrahl $d_{1}$ der Involution $J$ geschnitten und von dem andern $d_{2}$ berührt wird, so sind sämmtliche singulären Punkte von $\sigma$ reell; es fallen jedoch in dem Berührungspunkte von $d_{2}$ mit $C$ zwei Coincidenzpunkte und zwei Verzweigungspunkte mit einem Doppelpunkte von $\sigma$ und dem Berührungspunkte von $d_{2}$ mit $\Gamma$ zusammen. $C$ und $\Gamma$ durchschneiden einander in den beiden getrennten Verzweigungspunkten und berühren einander in 
dem gemeinsamen Berührungspunkte mit $d_{2}$. Der den Berïhrungspunkt enthaltende Theil von $C$ zwischen den Verzweigungspunkten liegt innerhalb $\Gamma$, ist somit parasitisch.

Berührt $C$ beide Doppelstrahlen, so reduciert sich das Punktsystem $\sigma$ auf die zwei Berührungspunkte; in jedem derselben rereinigen sich wie vorhin in dem einen die singulären Elemente. $\Gamma$ degeneriert hier in die beiden Doppelstrahlen von $J$.

18. Zum Sehlusse soll noch der Fall besprochen werden, der sich ergibt, wenn $C$ zwei einander entsprechende Strahlen $e$ und $e^{\prime}$ der hyperbolischen Involution $J$ ber ührt.

Die Berührungspunkte derselben sind die beiden Doppelpunkte $T_{1}, T_{2}$ des Punktsystems $\sigma$; mit ihnen fallen die Paare der nicht entsprechenden Verzweigungspunkte, $V_{3}, V_{4}$, bezw. $V_{1}, V_{2}$, zusammen. Da einer der beiden Doppelstrahlen, z. B. $d_{1}$, von $C$ gesehnitten werden muss, so ist das auf diesem gelegene Paar der Coincidenzpunkte $D_{1}, D_{1}^{\prime}$ reell, jenes auf $d_{2}$ hingegen imaginär.

Jedes zwischen $e$ und $e^{\prime}$ und in demselben Winkelraum mit $d_{1}$ gelegene Strahlenpaar $a, a^{\prime}$ der Involution $J$ schneidet auf $C$ ein Quadrupel reeller zusammengehöriger Punkte $A_{1}, A_{2} ; A_{1}^{\prime} A_{2}^{\prime}$ von $\sigma$ aus. Thre Verbindungsgeraden $A_{1} A_{2}^{\prime}, A_{2} A_{1}^{\prime} ; A_{1} A_{1}^{\prime}, A_{2} A_{2}^{\prime}$, welche Tangenten von $\Gamma$ sein müssen, gehen paarweise durch die Schnittpunkte $I, I I$ der Doppelstrahlen $d_{1}, d_{2}$ von $J$ mit der Polaren $s$ des Scheitels $S$ ron $J$ in Bezug auf $C$.

Dies erhellit daraus, dass je vier zusammengehörige Punkte von $\sigma$ die Ecken eines vollständigen Viereckes bilden, dessen Diagonalseiten die Doppelstrahlen $d_{1}$ und $d_{2}$ der Involution $J$ und die Polare $s$ von $S$ in Bezug auf $C$ sind. Es ist somit das Polardreieck $S I I l$ von $C$ das gemeinsame Diagonaldreieck aller jener Vierecke; daher gehen die Paare aller Gegenseiten, die nicht mit entsprechenden Strahlen ron $J$ zusammenfallen, durch die Diagonalecken $I$ und $I I$. In der letzteren treffen einander offenbar auch die Tangenten von $C$ in den Coincidenzpunkten $C_{1}$ und $C_{1}^{\prime}$.

Aus dem Voranstehenden folgt nun, dass der Directionskegelschnitt $\Gamma$ in diesem Falle in das Paar der Schnittpunkte $I$ und $I I$ der Doppelstrahlen von $J$ mit $s$ degeneriert.

Es ist daraus ferner zu erkennen, dass das durch die gegebene hyperbolische Strahleninvolution $J$ auf $C$ erzeugte Punktsystem $\sigma$ noch zwei weitere hyperbolische Strahleninvolutionen $J_{\mathrm{I}}$ and $J_{\text {II }}$ mit den Scheiteln $I$ und $I I$ bestimmt, deren entsprechende Strahlen die zweiten und dritten (nicht durch $S$ gehenden) Gegenseitenpaare der von den einzelnen Quadrupeln von $\sigma$ gebildeten vollständigen Vierecke sind..$^{x}$

1) Eine Vergleichung der obigen Erörterungen mit jenen in Art. 16 lässt die Übereinstimmung der hier gegebenen Involution $J$ mit der dort aus der gegebenen $J$ abgeleiteten $J^{\prime}$ erkennen, wobei an die Stelle der dortigen Strahlen $x, x^{\prime}$ und $d_{1}, d_{2}$ nun die Doppelstrahlen $d_{1}, d_{2}$, bezw. das Strahlenpaar $e, e^{\prime}$ treten und an die Stelle von $\mathbf{I}$ nun $C$; dann sind die Diagonaldreiecke $S X X^{\prime}$ und $S I I I$ und insbesondere auch die früheren Diagonalecken $X$ und $X^{\prime}$ mit den jetzigen $I$ und $I I$ identisch. 
Diese drei hyperbolischen Strahleninvolutionen $J, J_{I}, J_{I I}$, deren Scheitel die Ecken und deren Doppelstrahlen die Seiten des Polardreieckes SIII (i mallgemeinen eines beliebigen Polardreieckes) des Kegelschnittes $C$ sind, müssen offenbar projectivisch sein.

Je zwei derselben haben einen Doppelstrahl entsprechend gemein, liegen somit perspectiviseh${ }^{1}$ ), während dem zweiten Doppelstrahl der einen die Tangenten von $C$ aus dem Scheitel der andern entsprechen $C$.

Jezwei von den drei Involutionen erzeugen den Kegelschnitt $C$.

1) Je zwei können auch als projectivische Strahleninvolutionen zweiten Frades in reducierter Lage erster Ordnung bezeichnet werden. Der gemeinschaftliche Doppelstrahl ist als ein Theil des Erzeugnisses zu betrachten. Vgl. Weyr, "Beiträge zur Curvenlehre", pag. 50 und 52. 